PROCEEDINGS OF THE

AMERICAN MATHEMATICAL SOCIETY

Volume 135, Number 9, September 2007, Pages 2747-2750

S 0002-9939(07)08866-1

Article electronically published on May 9, 2007

\title{
FAILURE OF SEPARATION BY QUASI-HOMOMORPHISMS IN MAPPING CLASS GROUPS
}

\author{
H. ENDO AND D. KOTSCHICK
}

(Communicated by Daniel Ruberman)

\begin{abstract}
We show that mapping class groups of surfaces of genus at least two contain elements of infinite order that are not conjugate to their inverses, but whose powers have bounded torsion lengths. In particular every homogeneous quasi-homomorphism vanishes on such an element, showing that elements of infinite order not conjugate to their inverses cannot be separated by quasi-homomorphisms.
\end{abstract}

This note was motivated by the work of Polterovich and Rudnick [12, 13, who used the geometry of the hyperbolic plane to show that on $S L_{2}(\mathbb{Z})$ quasihomomorphisms exist in abundance and have interesting properties. One of their results is the following:

Theorem 1 ([12]). Let $g \in S L_{2}(\mathbb{Z})$ be an element of infinite order not conjugate to its inverse. Then there exists a homogeneous quasi-homomorphism $\varphi: S L_{2}(\mathbb{Z}) \rightarrow \mathbb{R}$ with $\varphi(g) \neq 0$.

Since homogeneous quasi-homomorphisms satisfy $\varphi\left(g^{-1}\right)=-\varphi(g)$ and are constant on conjugacy classes, the assumption of the theorem is clearly necessary 1

In view of Brooks's classical constructions of quasi-homomorphisms, this result is not suprising. In fact, Polterovich and Rudnick [12] pointed out that such a result essentially holds in much greater generality, because for all non-elementary Gromov hyperbolic groups it can be deduced from the work of Epstein and Fujiwara [5].

In [13, Polterovich and Rudnick generalized Theorem 10 to the following "separation theorem":

Theorem 2 ([13]). Let $g \in S L_{2}(\mathbb{Z})$ be a primitive element of infinite order not conjugate to its inverse, and $g_{1}, \ldots, g_{n} \in S L_{2}(\mathbb{Z})$ any finite number of elements not conjugate to any power of $g$. Then there exists a homogeneous quasi-homomorphism $\varphi: S L_{2}(\mathbb{Z}) \rightarrow \mathbb{R}$ with $\varphi(g) \neq 0$ and $\varphi\left(g_{1}\right)=\ldots=\varphi\left(g_{n}\right)=0$.

Thinking of $S L_{2}(\mathbb{Z})$ as the mapping class group of the two-torus, one naturally wonders whether Theorems 1 and 2 can be generalized to the mapping class groups

Received by the editors June 8, 2006.

2000 Mathematics Subject Classification. Primary 20F65; Secondary 20F12, 20F69, 57M07.

The second author would like to thank L. Polterovich for a conversation raising the question whether a separation theorem for mapping class groups of higher genus surfaces holds, and K. Fujiwara and J. McCarthy for useful comments. Support from the Deutsche Forschungsgemeinschaft and from JSPS Grant 18540083 is gratefully acknowledged.

${ }^{1}$ The reader can consult [8] for background on quasi-homomorphisms. 
of higher-genus surfaces. Several years ago we proved that there are non-trivial homogeneous quasi-homomorphisms on mapping class groups [4. Bestvina and Fujiwara [1] then showed that the space of such quasi-homomorphisms is infinitedimensional, and Polterovich asked whether it might be possible to prove a separation theorem in the spirit of Theorem 2 for mapping class groups. On the one hand, mapping class groups are perfect if the genus of the underlying surface is at least three [14, so they certainly have no homomorphisms to Abelian groups. On the other hand, they are residually finite [7, thus their elements can be separated by homomorphisms to finite groups, and by linear representations.

It is our purpose here to show that elements in mapping class groups cannot be separated by quasi-homomorphisms, by showing that the analogue of Theorem 1 and, a fortiori, the analogue of Theorem 2 fail for mapping class groups of surfaces of genus $\geq 2$. We shall prove the following:

Theorem 3. For every closed oriented surface of genus at least 2 there exist primitive elements $g$ of infinite order in its mapping class group of orientation-preserving diffeomorphisms such that $g^{k}$ is not conjugate to $g^{-k}$ for all $k \neq 0$, but all powers of $g$ are products of some fixed number of torsion elements.

It follows from the boundedness of the torsion lengths $t\left(g^{n}\right)$ that the stable torsion length

$$
\|g\|_{T}=\lim _{n \rightarrow \infty} \frac{t\left(g^{n}\right)}{n}
$$

vanishes, which, by the results of 8 , implies the vanishing of the stable commutator length. In fact, we will check explicitly that the powers of $g$ have bounded commutator lengths. The vanishing of the stable torsion length of $g$ also implies that every homogeneous quasi-homomorphism must vanish on $g$, by the estimate

$$
\|g\|_{T} \geq \frac{|\varphi(g)|}{D(\varphi)}
$$

where $D(\varphi)$ denotes the defect of $\varphi$; compare [8].

To put Theorem 3 into perspective, recall that, on the one hand, mapping class groups are not hyperbolic, for example because they contain Abelian subgroups of large ranks generated by Dehn twists along disjoint curves; cf. [2]. Reducible elements that are products of commuting Dehn twists, or have powers which are such products, are crucial to our construction, which is a further generalization of examples in [8], with roots in the work of McCarthy and Papadopoulos [1]. On the other hand, the construction of quasi-homomorphisms due to Bestvina and Fujiwara [1] does not require the group to be hyperbolic. All that is needed is a suitably weakly proper action on a $\delta$-hyperbolic space, and this weaker property holds for the actions of mapping class groups on complexes of curves, because of the work of Masur and Minsky [9]. Polterovich and Rudnick 13] suggest that the techniques of [1] might lead to a separation theorem for hyperbolic groups. Although we show that the separation theorem cannot hold for weakly hyperbolic groups like the mapping class groups, it is still possible that a separation theorem does hold if one restricts to pseudo-Anosov elements of mapping class groups, because only these act hyperbolically on the curve complex; see [9, 1]. Results in this direction are contained in forthcoming work of Calegari and Fujiwara 3. Their results are in contrast to Theorems 1 and 2, where $g$ is not assumed to be hyperbolic. 
Proof of Theorem 3. We denote by $\Sigma=\Sigma_{h}$ a fixed closed oriented surface of genus $h \geq 2$. Let $a, b$ and $c$ be disjoint non-separating simple closed curves on $\Sigma$ in distinct isotopy classes, and let $f=t_{a}^{-1} t_{b}^{-1} t_{c}^{2}$, where $t_{\alpha}$ denotes the right-handed Dehn twist along $\alpha$. Then $f^{k}=t_{a}^{-k} t_{b}^{-k} t_{c}^{2 k}$, for all integers $k$. In particular, $f$ is of infinite order in the mapping class group of $\Sigma$.

Now we use the following obvious lemma:

Lemma 4. Let $a$ and $b$ be non-separating disjoint simple closed curves on $\Sigma$. Then there exists an involution $\varphi: \Sigma \rightarrow \Sigma$ interchanging $a$ and $b$.

This allows us to find involutions $\varphi$ interchanging $a$ and $c$, and $\psi$ interchanging $b$ and $c$. Then

$$
f^{k}=t_{c}^{k} t_{a}^{-k} t_{c}^{k} t_{b}^{-k}=t_{c}^{k} \varphi\left(t_{c}^{k}\right)^{-1} \cdot \varphi \cdot t_{c}^{k} \psi\left(t_{c}^{k}\right)^{-1} \cdot \psi=\left[t_{c}^{k}, \varphi\right] \cdot\left[t_{c}^{k}, \psi\right] .
$$

Thus $f^{k}$ is both a product of four involutions and a product of two commutators. Therefore both the torsion length and the commutator length of $f^{k}$ are bounded independently of $k$. In particular, all homogeneous quasi-homomorphisms vanish on $f$.

Suppose now that $f^{k}$ and $f^{l}$ are conjugate for some $k$ and $l$. The system of curves consisting of $a, b$ and $c$ is a reducing system for all powers of $f$. In fact, in the terminology of Matsumoto and Montesinos [10, these three curves form a precise reducing system. As precise reducing systems are unique up to isotopy, a conjugacy between $f^{k}$ and $f^{l}$ must permute the isotopy classes of these three curves in such a way that the twisting numbers along the curves are matched up. But the triples of twisting or screw numbers are $(-k,-k, 2 k)$ and $(-l,-l, 2 l)$, so that we conclude $k=l$. In particular, $f$ cannot be conjugate to its inverse.

The element $f$ considered above is not always primitive, as it may be expressed as the square of $\alpha t_{a}^{-1} t_{c}$, for any involution $\alpha$ interchanging $a$ and $b$ and fixing $c$ (not necessarily pointwise). However, as the mapping class group of $\Sigma$ is residually finite [7, there exists a primitive element $g$ such that $f$ is a power of $g$. Then all powers of $g$ also have bounded torsion and commutator lengths, and every homogeneous quasi-homomorphism vanishes on $g$. If two distinct powers of $g$ were conjugate to each other, then the same would be true for $f$.

This completes the proof of Theorem 3 .

Remark 5. The ideas on reducing systems from [10] that we have used to show that distinct powers of $f$ are not conjugate to each other are quite classical, going back at least to Nielsen. Another modern approach to this topic is due to Birman, Lubotzky and McCarthy [2, and we could just as well base our argument on this reference.

Remark 6 . There are of course many other reducible mapping classes to which similar arguments apply. For example, assuming $h \geq 3$, we can find disjoint nonseparating non-isotopic simple closed curves $a_{1}, a_{2}, b_{1}$ and $b_{2}$ with the following property:

$$
\Sigma \backslash\left(a_{1} \cup a_{2}\right) \text { is connected, but } \Sigma \backslash\left(b_{1} \cup b_{2}\right) \text { is not. }
$$

By Lemma 4 there exist involutions $\varphi_{i}$ interchanging $a_{i}$ with $b_{i}$. The element $g=t_{a_{1}} t_{a_{2}} t_{b_{1}}^{-1} t_{b_{2}}^{-1}$ has the property that

$$
g^{k}=t_{a_{1}}^{k} t_{b_{1}}^{-k} t_{a_{2}}^{k} t_{b_{2}}^{-k}=t_{a_{1}}^{k} \varphi_{1} t_{a_{1}}^{-k} \cdot \varphi_{1} \cdot t_{a_{2}}^{k} \varphi_{2} t_{a_{2}}^{-k} \cdot \varphi_{2}=\left[t_{a_{1}}^{k}, \varphi_{1}\right] \cdot\left[t_{a_{2}}^{k}, \varphi_{2}\right],
$$

which is a product of 4 involutions, and also a product of 2 commutators. 
In this case the set of curves $a_{1}, a_{2}, b_{1}$ and $b_{2}$ forms a precise reducing system for every $g^{k}$, and the twisting numbers are $(k, k,-k,-k)$. Now if $g^{k}$ were conjugate to $g^{-k}$, then a conjugacy would have to interchange the isotopy classes of $a_{1} \cup a_{2}$ and of $b_{1} \cup b_{2}$, but this is impossible by $(\star)$.

Remark 7. If an element $g$ in a mapping class group can be expressed as a product of positive powers of Dehn twists along disjoint curves, then by [4, 8 , there is a homogeneous quasi-homomorphism $\varphi$ with $\varphi(g) \neq 0$. On the other hand, it follows from Lemma 4 that $t_{a} t_{b}^{-1}$ is conjugate to its inverse for any two disjoint nonseparating simple closed curves. Thus the examples we have given in the proof of Theorem 3 and in Remark 6 are the simplest reducible mapping classes which exhibit the failure of Theorems 1 and 2 for mapping class groups.

Remark 8. Our examples show that the linear growth of the commutator length for products of positive powers of Dehn twists along disjoint curves depends on restricting the chirality of the Dehn twists, even after excluding elements conjugate to their inverses. For linear growth of the word length no such restriction of the chirality is necessary [6].

\section{REFERENCES}

1. M. Bestvina and K. Fujiwara, Bounded cohomology of subgroups of mapping class groups, Geometry \& Topology 6 (2002), 69-89. MR1914565 (2003f:57003)

2. J. Birman, A. Lubotzky and J. McCarthy, Abelian and solvable subgroups of the mapping class group, Duke Math. J. 50 (1983), 1107-1120. MR.0726319 (85k:20126)

3. D. Calegari and K. Fujiwara, Stable commutator length in word hyperbolic groups, preprint, arXiv:math.gr/0611889 v2, 16 Jan 2007.

4. H. Endo and D. Kotschick, Bounded cohomology and non-uniform perfection of mapping class groups, Invent. math. 144 (2001), 169-175. MR.1821147 (2001m:57046)

5. D. Epstein and K. Fujiwara, The second bounded cohomology of word-hyperbolic groups, Topology 36 (1997), 1275-1289. MR.1452851 (98k:20088)

6. B. Farb, A. Lubotzky and Y. N. Minsky, Rank one phenomena in mapping class groups, Duke Math. J. 106 (2001), 581-597. MR1813237 (2001k:20076)

7. E. Grossman, On the residual finiteness of certain mapping class groups, J. London Math. Soc. 9 (1974), 160-164. MR0405423 (53:9216)

8. D. Kotschick, Quasi-homomorphisms and stable lengths in mapping class groups, Proc. Amer. Math. Soc. 132 (2004), 3167-3175. MR.2073290(2005e:20065)

9. H. A. Masur and Y. N. Minsky, Geometry of the complex of curves. I. Hyperbolicity, Invent. math. 138 (1999), 103-149. MR1714338(2000i:57027)

10. Y. Matsumoto and J. Montesinos-Amilibia, Pseudo-periodic homeomorphisms and degeneration of Riemann surfaces, Bull. Amer. Math. Soc. 30 (1994), 70-75. MR 1217354|(94h:30057)

11. J. McCarthy and A. Papadopoulos, Involutions in surface mapping class groups, Enseign. Math. 33 (1987), 275-290. MR0925990 (89a:57010)

12. L. Polterovich and Z. Rudnick, Kick stability in groups and dynamical systems, Nonlinearity 14 (2001), 1331-1363. MR1862824 (2003d:37003)

13. L. Polterovich and Z. Rudnick, Stable mixing for cat maps and quasi-morphisms of the modular group, Ergodic Theory Dynam. Systems 24 (2004), 609-619. MR2054053 (2005b:37043)

14. J. Powell, Two theorems on the mapping class group of a surface, Proc. Amer. Math. Soc. 68 (1978), 347-350. MR0494115 (58:13045)

Department of Mathematics, Graduate School of Science, Osaka University, ToyONAKA, OSAKA 560-0043, JAPAN

E-mail address: endo@math.wani.osaka-u.ac.jp

Mathematisches Institut, Ludwig-Maximilians-Universität München, Theresienstr. 39, 80333 MünChen, Germany

E-mail address: dieter@member.ams.org 